\title{
COVID-19 and Multiple Sclerosis: Predisposition and Precautions in Treatment
}

\author{
Shaghayegh Sadeghmousavi ${ }^{1,2} \cdot$ Nima Rezaei $^{3,4,5}$ (B)
}

Accepted: 31 August 2020 / Published online: 3 September 2020

(C) Springer Nature Switzerland AG 2020

\begin{abstract}
Infection with severe acute respiratory syndrome coronavirus 2 (SARS-CoV-2), leading to Coronavirus disease 2019 (COVID19), is not always confined to the respiratory tract, while patients with can develop neurological manifestations. The patients with multiple sclerosis (MS) pose challenges in this pandemic situation, because of the immunosuppressive medications they get and the fact that viral infections may contribute to MS exacerbation and relapses as an environmental factor in genetically predisposed individuals. Herein, possible consequences of COVID-19 which may carry for the MS patients and the underlying mechanisms of its impact are discussed.
\end{abstract}

Keywords SARS-CoV-2 $\cdot$ COVID-19 $\cdot$ Nervous system $\cdot$ Neurological symptoms $\cdot$ Multiple sclerosis

\section{Introduction}

In December 2019, an outbreak of respiratory disease due to a novel coronavirus $(\mathrm{CoV})$, named severe acute respiratory syndrome coronavirus 2 (SARS-CoV-2), occurred first in Wuhan, China; and then rapidly spread globally. The World Health Organization (WHO) named this disease coronavirus disease 2019 (COVID-19) [1-5]. In addition to the respiratory tract symptoms, COVID-19 patients can develop neurological symptoms, including dizziness, headache, impaired consciousness, acute cerebrovascular disease, encephalopathy,

This article is part of the Topical Collection on COVID-19

Nima Rezaei

rezaei_nima@tums.ac.ir

1 Neuroimaging Network (NIN), Universal Scientific Education and Research Network (USERN), Tehran, Iran

2 School of Medicine, Shahid Beheshti University of Medical Sciences, Tehran, Iran

3 Research Center for Immunodeficiencies, Pediatrics Center of Excellence, Children's Medical Center Hospital, Tehran University of Medical Sciences, Dr Qarib St, Keshavarz Blvd, Tehran 14194, Iran

4 Department of Immunology, School of Medicine, Tehran University of Medical Sciences, Tehran, Iran

5 Network of Immunity in Infection, Malignancy and Autoimmunity (NIIMA), Universal Scientific Education and Research Network (USERN), Tehran, Iran ataxia, seizure, peripheral nervous system involvement (taste, smell, and vision impairment such as optic neuritis, and Guillain-Barré syndrome), and nerve pain [6-14]. Also, based on autopsy studies, the presence of brain tissue edema and partial neuronal degeneration was confirmed in these patients [15].

One of the neurological involvements of $\mathrm{CoVs}$ that has been reported is their implication in chronic neuroinflammatory diseases, including multiple sclerosis (MS) [16-19]. In this study, we aimed to review the research into the impacts of COVID-19 on MS.

\section{MS in COVID-19}

Multiple sclerosis (MS) is a chronic immune-mediated and neurodegenerative disorder that can be developed by both genetic and environmental factors [20]. The major symptoms are sensory, visual, and motor impairments, fatigue, pain, and cognitive deficits [21]. The presence of sclerotic plaque is the hallmark of this demyelinating disease, which indicates the final stage of a process including the inflammation, demyelination and remyelination, oligodendrocyte depletion and astrocytosis, and neuronal and axon degeneration [21, 22].

The pathology of this disease starts with increased migration of autoreactive lymphocytes across the blood-brain barrier. Regulatory defects allow these cells to transit from physiological states to a pathological cascade and set up an immune response within the brain. Regulatory lymphocytes in MS 
patients fail to suppress effector cells [21, 23]. This local failure in regulatory mechanism in the brain leads to the inflammation in particular sites due to the accumulation of perivascular CD8+ cells and constructing plaques around the lateral ventricles and corpus callosum, in the cortex and subcortical white matter, the optic nerves and brainstem, and the spinal cord [21]. With the accumulation of T and B lymphocytes, plasma cells, and macrophages, pro-inflammatory cytokines amplify the immune response through naive microglia [24-28]. B-lymphoid follicles in the meninges start the humoral immune response that can damage the nearby cortex due to intrathecal antibody production [29].

The environmental factors in addition to genetic risk factors can participate in disease development $[30,31]$. Microbial infections as triggers can lead to autoimmunity and clinical expression of MS in genetically predisposed individuals [32-35]. Studies have suggested a robust relationship between viral infections and exacerbations of MS [36-45]. Buljevac et al. assessed the contribution of systemic infections to the course of exacerbations in 73 patients with relapsing-remitting MS. They found that there was an increased risk of exacerbations (rate ratio 2.1) around the time of an infection especially an upper airway infection in comparison with the group without a clinical infection [37]. In another study, Sibley et al. assessed environmental factors that have roles in exacerbation or progression of the MS in patients with MS at monthly intervals over 8 years. Annual exacerbation rates in those patients that had infection were almost threefold greater than those who were not at risk [38]. Anderson et al. recorded prospectively upper respiratory, gastrointestinal infections and serological diagnosis of five common viral infections in 60 benign multiple sclerosis patients. During 4-week at risk (AR) periods encompassing common infections, a significant excess of MS relapses was found in the AR period, with a relative risk of 1.3 [39]. In another study, Oikonen et al. studied the effect of environmental viral infections on MS relapse occurrence and compared retrospectively the monthly relapse occurrence of $407 \mathrm{MS}$ patients. They suggested that after a peak in diagnosed influenza A cases in the general population, the MS relapse occurrence was 6.5 times higher (95\% CI 1.8-24.0) and also a rise in MS relapse followed Epstein-Barr virus (EBV) infections (OR 4.4, 95\% CI 1.3-15.1) [40].

During this rapid and global spread of COVID-19, a higher risk for severe disease with acute respiratory failure, intensive care unit admission, and/or death is considered for patients with comorbidities [46].

The concerns for patients with MS are because of an increased risk of infection with severe acute respiratory syndrome coronavirus 2 (SARS-CoV-2) and more severe disease due to taking disease-modifying therapies that have immunosuppressive effects in MS patients [47, 48] and also the fact that viral infections may contribute to MS exacerbation and relapses as an environmental factor in genetically predisposed individuals $[38,39]$.

\section{Immunosuppressive Therapies in MS}

Considering the presented challenge to MS patients by this pandemic, Moss et al. described the influence of COVID-19 on routine MS management in 3028 participants. Most of the participants continued DMT treatments and had no intentional delays or discontinuations, but $5 \%$ of survey responders had changed their therapy plans, especially delays in infusions due to their healthcare provider's advice or other reasons including their concerns about treatment during the COVID-19 pandemic or due to interruptions of care (15.5\%). Their findings also demonstrate that younger people with lower socioeconomic status may be at higher risk for exposure and should be considered for educational intervention and clinician support to lower exposure [49].

Immunosuppressive therapies for MS might confer additional risks against COVID-19; however, taking immunosuppressive treatments was not associated with a significantly increased risk of hospitalization or fatal outcome, according to the published case reports. Parrotta et al. reported outcomes of coronavirus on 76 patients with MS; $84 \%$ of patients were on disease-modifying therapy (DMT). The most common COVID-19 symptoms were fever and cough, but $21.1 \%$ of patients had neurologic symptom recrudescence preceding or coinciding with the infection. A total of $1823.7 \%$ were hospitalized; $10.5 \%$ had COVID-19 critical illness or related death. Those hospitalized or with critical illness or death were older age, had presence of comorbidities, progressive disease, and a nonambulatory status. As a result, DMT usage did not emerge as a predictor of poor COVID-19 outcomes [50]. In another study, Dalla Costa et al. assessed the impact of MS treatments on COVID-19 risk separately for each drug in 399 patients in Italy, Spain, and Denmark by using remote monitoring technologies. According to the results, there was no evidence of a worse COVID-19 evolution in patients affected by MS; however, among DMTs, immune-resetting drugs including alemtuzumab and cladribine changed the risk and severity of COVID-19 in comparison with injectables [51]. Also, Novi et al. reported on a patient treated with ocrelizumab for primary progressive MS who developed COVID-19. Despite complete B cell depletion leading to ocrelizumab, patient symptoms abated a few days after hospitalization and were discharged to home quarantine, and after 14 days, no new symptoms occurred. So, despite the role of immunosuppression treatment in exposing infected patients to even higher risks of disease complications, they declared that immunosuppressants can be tested as a potential treatment for COVID-19 [52]. Also, Kloc et al. suggested the protective 
role of MS DMTs against acute respiratory distress syndrome (ARDS) in COVID-19 patients. They found that clinically approved MS drugs, fingolimod and siponimod, are potent in inhibition of ACE2 receptors expression and the recruitment of macrophages to the lungs of these patients, which can lower cytokine storm and attenuate ARDS [53].

Zheng et al. in a review study discussed the safety of each DMT that can be used during the COVID-19 pandemic. They demonstrated that conventional injectable medications such as natalizumab (but its monthly intravenous administration may increase the exposure risk) because of their safest immune profile can be used more frequently in mild MS patients during the pandemic, and the non-cell-depleting oral agents are likely safer than cell-depleting agents and are safer than all intravenous agents from the exposure risk standpoint. They have mentioned that among the cell-depleting DMTs, ocrelizumab is probably the safest if can be used sparingly, and cladribine may be relatively safer than alemtuzumab in the oral form. In the end, they suggest that neuroimmunologists should consider the exact mechanism of action of each DMT and their interactions with the immune response against SARSCoV-2 and its future vaccine [54]. Despite the results of above-mentioned studies, another study by Crescenzo et al. suggested the prevalence and severity of COVID-19 in 1034 MS patients in Italy; the prevalence of COVID-19 seemed to be much higher (2.5-fold) than the general population [55].

Another issue that should be noted in this population is the effect of DMTs on immune responses to SARS-CoV-2 vaccinations $[54,56]$. An effective immune response to vaccination is provided by the adaptive immune system including B cells and $\mathrm{T}$ cells. With vaccination, leading to the presence of their target antigen, $\mathrm{B}$ and $\mathrm{T}$ cells clonally expand with some transforming into memory cells and also proliferate into effector cells after re-exposure to their target antigen [57]. Leading immunomodulatory and immunosuppressive effects of different DMTs may sophisticate the condition of vaccinations. Live vaccines are generally contraindicated in MS patients on immunosuppressive treatments. Also, DMTs that affect the adaptive immune system can lower the vaccines efficacy by impairing the development of long-term immune memory [56]. Ciotti et al. in a review suggested the impact of DMTs for MS on vaccine responses in humans and concluded that apart from beta-interferons, many MS DMTs impair humoral immune responses to a variety of vaccine types [56].

Due to these contradictory results, further studies are needed to clarify the relation between COVID-19 severity/risk and immunosuppressive medications.

\section{COVID-19 in MS Relapses}

Interestingly in a study by Stojanov et al, the influence of COVID-19 pandemic on the psychological status of patients with relapsing-remitting multiple sclerosis (RRMS) was assessed (in 95 RRMS patients). Results on all questionnaires were worse in RRMS patients than in healthy individuals $(p<0.01)$. The main concerns of these patients are the following: (1) that their disease could worsen due to COVID-19 $(36.4 \%)$, (2) difficulties in drug availability (43.6\%), and (3) that they could not access the hospital as previously usual condition (72.4\%). So they suggested that healthcare organizations should consider professional therapeutic advice and psychosocial support for this population of patients during the pandemic [58].

Although association of other types of coronaviruses to demyelinating diseases has been reported, a connection between SARS-CoV-2 and MS exacerbation has been reported in few studies [19]. For example in a study by Palao et al., a patient with decreased visual acuity after being infected by Covid-19 was reported. The patient had previously experienced no neurological symptoms. The MRI suggested inflammation in the right optic nerve and demyelinating lesions in the CNS. They suggested that in attention to the matching of the diagnosis criteria of MS and presence of non-enhancing periventricular lesions, SARS-CoV-2 may have acted as a precipitating factor and the MS pathogenic process had already started before the infection [14].

The mechanisms which may lead COVID-19 to trigger an exacerbation of MS in these patients could be as follows:

A. Toll-like receptors (TLRs) have roles in host defense and the recognition of invading pathogens and involve in MS pathophysiology. These receptors recognize viral particles and contribute to the modification of the immune response in MS patients. This can associate viral infections, including coronavirus, to the development of demyelinating diseases [59].

B. The typical MS lesions are associated with various degrees of inflammatory cells. Also, COVID-19 is associated with alterations in immune cell populations and the respective T cell, B cell, and NK-cell subsets. So, MS relapses can occur due to the initiation of the responses of the innate and the adaptive immune system by the virus [60].

C. Another factor that can relate coronavirus to MS relapsing is the associated cytokine storms of severe COVID19. This neuroinflammation especially in the presence of disrupted blood-brain barrier (BBB) integrity can lead to the exacerbation of MS [61].

D. Direct CNS invasion can play a role in the relapses. It has been shown that SARS-CoV-2 can invade the CNS and induce direct neuron loss [17, 62]. The presence of the ACE2 receptor in tissues determines viral cellular tropism in humans. It expresses widely throughout the CNS $[63,64]$. The presence of SARS-CoV-2 in the brain of MS patients involves in the pathogenesis MS and predisposes patients toward neurodegeneration $[61,65]$. So, 
a direct viral toxic mechanism can cause cellular damage to infected neural cells [66].

E. The demyelinating reaction is considered one of the complications of severe COVID-19 [6]. Since MS is a demyelinating disease, this pathology should be considered.

Based on the aforementioned studies, MS patients are at greater risk of COVID-19 infection and also disease exacerbations and relapses due to this viral infection. So, within the context of the ongoing COVID-19 crisis, the best management strategies should be considered for MS patients.

\section{Conclusion}

Multiple Sclerosis is a chronic inflammatory disease of the CNS with an autoimmune etiology [20]. The pathology of this disease starts with increased migration of autoreactive lymphocytes across the BBB. Regulatory defects allow these cells to transit from physiological states to a pathological cascade. Both genetic and environmental factors in MS patients can cause this impairment of the regulatory lymphocytes functions to suppress effector cells [21,23]. Microbial infections can act as triggers for inducing autoimmunity and clinical disease manifestations in genetically predisposed individuals [32-35]. Studies have suggested a robust relationship between viral infections and exacerbations of MS [36-45]. Based on the aforementioned studies, COVID-19 carries additional reasons for concern because of taking immunosuppressive therapies in MS patients $[47,48]$ and also the fact that viral infections may contribute to MS exacerbation and relapses as an environmental factor $[38,39]$. This study provides some insights regarding the impact of COVID-19 disease on actively treated patients with MS.

For clarification of the exact relationship between COVID19 and MS, further studies are needed. Meanwhile, clinicians should take care of patients with MS during the pandemic regarding the possible reciprocal association between the MS and COVID-19.

\section{Compliance with Ethical Standards}

Conflict of Interests The authors declare that they have no conflict of interest.

\section{References}

1. Zhu N, Zhang D, Wang W, Li X, Yang B, Song J, et al. A Novel Coronavirus from Patients with Pneumonia in China, 2019. N Engl J Med. 2020;382(8):727-33.
2. Zhou P, Yang X-L, Wang X-G, Hu B, Zhang L, Zhang W, et al. A pneumonia outbreak associated with a new coronavirus of probable bat origin. Nature. 2020;579(7798):270-3.

3. World Health Organization. Coronavirus disease (COVID-19) pandemic 2020 [July 09, 2020]. Available from: https://www.who.int/ emergencies/diseases/novel-coronavirus-2019.

4. Hanaei S, Rezaei N. COVID-19: developing from an outbreak to a pandemic. Arch Med Res. 2020. https://doi.org/10.1016/j.arcmed. 2020.04.021.

5. Jabbari P, Jabbari F, Ebrahimi S, Rezaei N. COVID-19: a chimera of two pandemics. Disaster Med Public Health Prep. 2020;1-2.

6. Mao L, Jin H, Wang M, Hu Y, Chen S, He Q, et al. Neurologic manifestations of hospitalized patients with coronavirus disease 2019 in Wuhan, China. JAMA Neurol. 2020;77(6):683-90.

7. Giacomelli A, Pezzati L, Conti F, Bernacchia D, Siano M, Oreni L, et al. Self-reported olfactory and taste disorders in patients with severe acute respiratory coronavirus 2 infection: a cross-sectional study. Clin Infect Dis. 2020;71:889-90.

8. Filatov A, Sharma P, Hindi F, Espinosa PS. Neurological Complications of Coronavirus Disease (COVID-19): Encephalopathy. Cureus. 2020;12(3):e7352-e.

9. Pleasure SJ, Green AJ, Josephson SA. The spectrum of neurologic disease in the severe acute respiratory syndrome coronavirus 2 pandemic infection: neurologists move to the frontlines. JAMA Neurol. 2020;77(6):679-80.

10. Jahanshahlu L, Rezaei N. Central Nervous System Involvement in COVID-19. Arch Med Res. 2020;S0188-4409(20)30797-9.

11. Saleki K, Banazadeh M, Saghazadeh A, Rezaei N. The involvement of the central nervous system in patients with COVID-19. Rev Neurosci. 2020;31(4):453-6.

12. Sedaghat $Z$, Karimi N. Guillain Barre syndrome associated with COVID-19 infection: a case report. J Clin Neurosci. 2020;76: 233-5.

13. Padroni M, Mastrangelo V, Asioli GM, Pavolucci L, AbuRumeileh S, Piscaglia MG, et al. Guillain-Barré syndrome following COVID-19: new infection, old complication?. J Neurol. 2020;267(7):1877-9.

14. Palao M, Fernández-Díaz E, Gracia-Gil J, Romero-Sánchez C, Díaz-Maroto I, Segura T. Multiple sclerosis following SARSCoV-2 infection. Mult Scler Relat Disord. 2020;45:102377.

15. Xu Z, Shi L, Wang Y, Zhang J, Huang L, Zhang C, et al. Pathological findings of COVID-19 associated with acute respiratory distress syndrome. Lancet Respir Med. 2020;8(4):420-2.

16. Pérez CA. Looking ahead: the risk of neurologic complications due to COVID-19. Neurology. 2020. https://doi.org/10.1212/CPJ. 0000000000000836.

17. Desforges M, Le Coupanec A, Dubeau P, Bourgouin A, Lajoie L, Dubé $\mathrm{M}$, et al. Human coronaviruses and other respiratory viruses: underestimated opportunistic pathogens of the central nervous system? Viruses. 2020;12(1):14.

18. Cristallo A, Gambaro F, Biamonti G, Ferrante P, Battaglia M, Cereda PM. Human coronavirus polyadenylated RNA sequences in cerebrospinal fluid from multiple sclerosis patients. New Microbiol. 1997;20(2):105-14.

19. Montalvan V, Lee J, Bueso T, De Toledo J, Rivas K. Neurological manifestations of COVID-19 and other coronavirus infections: a systematic review. Clin Neurol Neurosurg. 2020;194:105921.

20. Dendrou CA, Fugger L, Friese MA. Immunopathology of multiple sclerosis. Nat Rev Immunol. 2015;15(9):545-58.

21. Compston A, Coles A. Multiple sclerosis. Lancet (London, England). 2008;372(9648):1502-17.

22. Barres BA, Hart IK, Coles HS, Burne JF, Voyvodic JT, Richardson WD, et al. Cell death and control of cell survival in the oligodendrocyte lineage. Cell. 1992;70(1):31-46. 
23. Viglietta V, Baecher-Allan C, Weiner HL, Hafler DA. Loss of functional suppression by CD4+CD25+ regulatory T cells in patients with multiple sclerosis. J Exp Med. 2004;199(7):971-9.

24. Zajicek JP, Wing M, Scolding NJ, Compston DA. Interactions between oligodendrocytes and microglia. A major role for complement and tumour necrosis factor in oligodendrocyte adherence and killing. Brain J Neurol. 1992;115(Pt 6):1611-31.

25. Pérez-Cerdá F, Sánchez-Gómez MV, Matute C. The link of inflammation and neurodegeneration in progressive multiple sclerosis. Mult Scler Demyelinat Disord. 2016;1(1):1-8.

26. Milo R, Korczyn AD, Manouchehri N, Stüve O. The temporal and causal relationship between inflammation and neurodegeneration in multiple sclerosis. Mult Scler J. 2020;26(8):876-86.

27. Carstensen M, Christensen T, Stilund M, Møller HJ, Petersen EL, Petersen T. Activated monocytes and markers of inflammation in newly diagnosed multiple sclerosis. Immunol Cell Biol. 2020;98(7):549-62.

28. Stilund M, Gjelstrup MC, Petersen T, Møller HJ, Rasmussen PV, Christensen T. Biomarkers of inflammation and axonal degeneration/damage in patients with newly diagnosed multiple sclerosis: contributions of the soluble $\mathrm{CD} 163 \mathrm{CSF} /$ serum ratio to a biomarker panel. PLoS One. 2015;10(4):e0119681.

29. Magliozzi R, Howell O, Vora A, Serafini B, Nicholas R, Puopolo $\mathrm{M}$, et al. Meningeal B-cell follicles in secondary progressive multiple sclerosis associate with early onset of disease and severe cortical pathology. Brain J Neurol. 2007;130(Pt 4):1089-104.

30. Olsson T, Barcellos LF, Alfredsson L. Interactions between genetic, lifestyle and environmental risk factors for multiple sclerosis. Nat Rev Neurol. 2017;13(1):25-36.

31. Abbasi M, Nabavi SM, Fereshtehnejad SM, Jou NZ, Ansari I, Shayegannejad V, et al. Multiple sclerosis and environmental risk factors: a case-control study in Iran. Neurol Sci. 2017;38(11):194151.

32. Venkatesan A, Johnson RT. Infections and multiple sclerosis. Handb Clin Neurol. 2014;122:151-71.

33. Brown C. Aetiology: neighbourhood watch. Nature. 2016;540(7631):S4-6.

34. Najafi S, Ghane M, Yousefzadeh-Chabok S, Amiri M. The high prevalence of the varicella zoster virus in patients with relapsingremitting multiple sclerosis: a case-control study in the north of Iran. Jundishapur J Microbiol. 2016;9(3):e34158.

35. Yoshimura S, Isobe N, Matsushita T, Masaki K, Sato S, Kawano Y, et al. Genetic and infectious profiles influence cerebrospinal fluid IgG abnormality in Japanese multiple sclerosis patients. PLoS One. 2014;9(4):e95367.

36. Correale J, Fiol M, Gilmore W. The risk of relapses in multiple sclerosis during systemic infections. Neurology. 2006;67(4):652-9.

37. Buljevac D, Flach HZ, Hop WC, Hijdra D, Laman JD, Savelkoul HF, et al. Prospective study on the relationship between infections and multiple sclerosis exacerbations. Brain J Neurol. 2002;125(Pt 5):952-60.

38. Sibley W, Bamford C, Clark K. Clinical viral infections and multiple sclerosis. Lancet. 1985;325(8441):1313-5.

39. Andersen O, Lygner P-E, Bergström T, Andersson M, Vablne A. Viral infections trigger multiple sclerosis relapses: a prospective seroepidemiological study. J Neurol. 1993;240(7):417-22.

40. Oikonen M, Laaksonen M, Aalto V, Ilonen J, Salonen R, Erälinna J-P, et al. Temporal relationship between environmental influenza $\mathrm{A}$ and Epstein-Barr viral infections and high multiple sclerosis relapse occurrence. Mult Scler J. 2011;17(6):672-80.

41. Merelli E, Casoni F. Prognostic factors in multiple sclerosis: role of intercurrent infections and vaccinations against influenza and hepatitis B. Neurol Sci. 2000;21(2):S853-S6.
42. Marrodan M, Alessandro L, Farez MF, Correale J. The role of infections in multiple sclerosis. Mult Scler J. 2019;25(7):891-901.

43. Donati D. Viral infections and multiple sclerosis. Drug Discov Today Dis Model. 2020. https://doi.org/10.1016/j.ddmod.2020.02. 003.

44. Jakimovski D, Weinstock-Guttman B, Ramanathan M, Dwyer MG, Zivadinov R. Infections, vaccines and autoimmunity: a multiple sclerosis perspective. Vaccines. 2020;8(1):50.

45. Ryder E, Steelman AJ. Does upper respiratory infection exacerbate symptoms of multiple sclerosis? Future Microbiol. 2018;13(5): 503-5.

46. Devogelaere J, D'hooghe MB, Vanderhauwaert F, D'haeseleer M. Coronavirus disease 2019: favorable outcome in an immunosuppressed patient with multiple sclerosis. Neurol Sci. 2020;41:19813.

47. Willis M, Robertson N. Multiple sclerosis and the risk of infection: considerations in the threat of the novel coronavirus, COVID-19/ SARS-CoV-2. J Neurol. 2020;267(5):1567-9.

48. Amor S, Baker D, Khoury SJ, Schmierer K, Giovanonni G. SARSCoV-2 and multiple sclerosis: not all immune depleting DMTs are equal or bad. Ann Neurol. 2020;87:794-7.

49. Moss BP, Mahajan KR, Bermel RA, Hellisz K, Hua LH, Hudec T, et al. Multiple sclerosis management during the COVID-19 pandemic. Mult Scler. 2020;1352458520948231.

50. Parrotta E, Kister I, Charvet L, Sammarco C, Saha V, Charlson RE, et al. COVID-19 outcomes in MS. Observational study of early experience from NYU Multiple Sclerosis Comprehensive Care Center. Neurol Neuroimmunol Neuroinflamm. 2020;7(5):e835.

51. Dalla Costa G, Leocani L, Montalban X, Guerrero AI, Sørensen PS, Magyari M, et al. Real-time assessment of COVID-19 prevalence among multiple sclerosis patients: a multicenter European study. Neurol Sci. 2020;41:1647-50.

52. Novi G, Mikulska M, Briano F, Toscanini F, Tazza F, Uccelli A, et al. COVID-19 in a MS patient treated with ocrelizumab: does immunosuppression have a protective role? Mult Scler Relat Disord. 2020;42:102120.

53. Kloc M, Ghobrial RM. The multiple sclerosis (MS) drugs as a potential treatment of ARDS in COVID-19 patients. Mult Scler Relat Disord. 2020;45:102437.

54. Zheng C, Kar I, Chen CK, Sau C, Woodson S, Serra A, et al. Multiple sclerosis disease-modifying therapy and the COVID-19 pandemic: implications on the risk of infection and future vaccination. CNS Drugs. 2020;34(9):879-6.

55. Crescenzo F, Marastoni D, Bovo C, Calabrese M. Frequency and severity of COVID-19 in multiple sclerosis: a short single-site report from northern Italy. Mult Scler Relat Disord. 2020;44:102372.

56. Ciotti JR, Valtcheva MV, Cross AH. Effects of MS diseasemodifying therapies on responses to vaccinations: a review. Mult Scler Relat Disord. 2020;45:102439.

57. Clem AS. Fundamentals of vaccine immunology. J Global Infect Dis. 2011;3(1):73-8.

58. Stojanov A, Malobabic M, Milosevic V, Stojanov J, Vojinovic S, Stanojevic G, et al. Psychological status of patients with relapsingremitting multiple sclerosis during coronavirus disease-2019 outbreak. Mult Scler Relat Disord. 2020;45:102407.

59. Duffy L, O'Reilly SC. Toll-like receptors in the pathogenesis of autoimmune diseases: recent and emerging translational developments. ImmunoTargets Ther. 2016;5:69.

60. Kurtzke JF. Epidemiologic evidence for multiple sclerosis as an infection. Clin Microbiol Rev. 1993;6(4):382-427.

61. Boziki MK, Mentis A-FA, Shumilina M, Makshakov G, Evdoshenko E, Grigoriadis N. COVID-19 immunopathology and the central nervous system: implication for multiple sclerosis and 
other autoimmune diseases with associated demyelination. Brain Sci. 2020;10(6):345.

62. Wu Y, Xu X, Chen Z, Duan J, Hashimoto K, Yang L, et al. Nervous system involvement after infection with COVID-19 and other coronaviruses. Brain Behav Immun. 2020;87:18-22.

63. Doobay MF, Talman LS, Obr TD, Tian X, Davisson RL, Lazartigues E. Differential expression of neuronal ACE2 in transgenic mice with overexpression of the brain renin-angiotensin system. Am J Phys Regul Integr Comp Phys. 2007;292(1):R373-R81.

64. Xia H, Lazartigues E. Angiotensin-converting enzyme 2 in the brain: properties and future directions. J Neurochem. 2008;107(6):1482-94.
65. Iroegbu JD, Ifenatuoha $\mathrm{CW}$, Ijomone $\mathrm{OM}$. Potential neurological impact of coronaviruses: implications for the novel SARS-CoV-2. Neurol Sci. 2020;41(6):1329-37.

66. Lucchinetti C, Brück W, Parisi J, Scheithauer B, Rodriguez M, Lassmann H. Heterogeneity of multiple sclerosis lesions: implications for the pathogenesis of demyelination. Ann Neurol. 2000;47(6):707-17.

Publisher's Note Springer Nature remains neutral with regard to jurisdictional claims in published maps and institutional affiliations. 